\title{
Organização de Práticas de Saúde Equânimes em Atenção Primária em Região Metropolitana no Contexto dos Processos de Inclusão e Exclusão Social
}

\section{Organization of Equanimous Practices in Primary Health Attention within the Metropolitan Area with Respect to Social Inclusion and Exclusion Processes}

\author{
Nivaldo Carneiro Junior \\ Médico sanitarista, professor do Departamento de Medicina So- \\ cial da Faculdade de Ciências Médicas da Santa Casa de São Paulo \\ e Diretor do Centro de Saúde Escola Barra Funda Dr. Alexandre \\ Vranjac, Irmandade da Santa Casa de Misericórdia de São Paulo. \\ E-mail: nicarneirojrøuol.com.br \\ Marta Campagnoni Andrade \\ Médica sanitarista e pesquisadora do Centro de Saúde Escola \\ Barra Funda Dr. Alexandre Vranjac, Irmandade da Santa Casa \\ de Misericórdia de São Paulo.

\section{Carla Gianna Luppi} \\ Médica sanitarista, professora do Departamento de Medicina \\ Social da Faculdade de Ciências Médicas da Santa Casa de São \\ Paulo e do Centro de Saúde Escola Barra Funda Dr. Alexandre \\ Vranjac, Irmandade da Santa Casa de Misericórdia de São Paulo. \\ E-mail: cgluppiळyahoo.com.br

\section{Cássio Silveira} \\ Sociólogo, professor do Departamento de Medicina Social da \\ Faculdade de Ciências Médicas da Santa Casa de São Paulo. \\ E-mail: cassio.silveiraœfcmscsp.edu.br
}

\section{Resumo}

Esse artigo apresenta uma experiência de organização da atenção primária em saúde norteada pelo conceito de eqüidade, entendida no contexto da justiça social, para grupos populacionais em situação de exclusão social na região central da cidade de São Paulo. As transformações sociais observadas na região são complexas, marcadas pela heterogeneidade dos grupos populacionais nos processos de exclusão e inclusão social. A inclusão por meio das políticas sociais é uma questão essencial que leva às chamadas políticas de integração, orientadas para a diminuição das desigualdades sociais. É necessário organizar processos de trabalho que dêem visibilidade a esses grupos sociais e incorporem na atenção as suas demandas, articulando-as com outras práticas sociais. Os serviços de atenção primária em saúde são ideais nessa perspectiva. Assim, o Centro de Saúde Escola Barra Funda organizou processos de trabalho visando dar visibilidade a esses segmentos populacionais e atenção diferenciada, pactuando no conjunto de suas ações. Nesse processo, alguns grupos tornaram-se visíveis e inclusos no cuidado à saúde: a população em situação de rua; as profissionais do sexo da região da Luz; os migrantes bolivianos e os moradores de favela. 0 serviço incorporou como parte de sua tecnologia o cuidado à saúde de grupos diferenciados da população em geral, particularizando-o, criando "portas de entrada" específicas, promovendo, desse modo, a eqüidade no acesso. Desafios conceituais e metodológicos se colocam na consolidação dessas práticas, requerendo 
análises dos processos de exclusão e inclusão social e nos modos do cuidado em saúde, tais como a integralidade e a autonomia dos sujeitos.

Palavras-chave: Inclusão e exclusão social; Eqüidade em saúde; Atenção primária em saúde; Atenção em saúde; Tecnologia em saúde.

\section{Abstract}

This article presents an organization of primary health attention guided by the concept of equality, within the social justice context, for population groups in social exclusion situations in the central region of the city of Sao Paulo. The social transformations observed in the region are complex, marked by the heterogeneity of the population groups within the social inclusion/exclusion processes. Inclusion through social policies is an essential item of integration policies, guided by the need to decrease social inequalities. It is necessary to organize work processes that give visibility to these social groups and incorporate their demands into health care, linking them to other social practices. For this aim, primary health care services are ideal. Barra Funda Educational Health Center organized work processes to provide visibility and differentiated attention to these segments. During this process, some groups became visible and were included in health care: the street population; the sex professionals in the region of Luz; the Bolivian immigrants and those living in shantytowns. As part of its technology, the service incorporated the health care of differentiated groups of the population in general, individualizing them, creating specific "entrance doorways", and promoting equanimity in access. Conceptual and methodological challenges were employed in the consolidation of these practices, which required analyses of the social inclusion/exclusion processes, and of the styles of health care, such as integrality and autonomy of the subjects.

Key words: Social Inclusion/Exclusion; Equanimity in Health; Primary Health Care; Health Technology.

\section{Introdução}

As transformações sociais observadas na cidade de São Paulo são muitas e variadas, em suas características, particularmente em relação à área central da cidade. Nessa área, encontram-se os mais variados segmentos da população: trabalhadores do setor formal e do informal da economia, que transitam na área central e ali constroem suas vidas; moradores locais com condições adequadas de vida; outros, que vivem em situação de precariedade, como os moradores de cortiços, favelas e imóveis ocupados; profissionais do sexo, imigrantes de outros países, indocumentados e, por fim, a população em situação de rua, que a presença nos logradouros escancara a verdadeira face da metrópole, que exclui os sujeitos das relações de trabalho e os impulsiona, cada vez mais, a processos complexos de exclusão social.

Essa complexidade, marcada pela heterogeneidade dos grupos sociais, tanto no que se refere às suas condições socioeconômicas como aos seus ciclos de vida (crianças, jovens, adultos e idosos), requer dos serviços públicos uma organização também complexa e dinâmica, que atenda às diferentes necessidades sentidas na área central.

Estruturar serviços públicos, em particular os de saúde, requer políticas, um conjunto de práticas, que supram as necessidades desses vários grupos e uma capacidade de refletir sobre a condição desses segmentos sociais, objetivando a efetividade e a eficácia social. Dessa forma, os processos de exclusão e inclusão social devem ser pensados e, a partir disso, podem ser organizadas práticas no interior dos serviços públicos nas diversas áreas sociais, com a criação de novas formas de articulação em rede, transformando as organizações técnico-administrativas de modo que elas possam incorporar esses segmentos populacionais em suas necessidades e demandas (Carneiro Junior; Silveira, 2003).

O debate sobre a inclusão de segmentos sociais vulneráveis nos serviços de saúde pressupõe minimamente, por um lado, definições mais precisas sobre o posicionamento desses sujeitos no conjunto das relações sociais, sejam elas relações primárias, cuja estrutura via de regra está assentada na unidade familiar, ou outras formas gregárias de relacionamento, como os imigrantes bolivianos, que dividem o espaço 
doméstico e de trabalho entre grupos de famílias; por outro lado, torna-se urgente a incorporação do debate sobre os processos sociais, que conduzem os sujeitos a processos de vulnerabilidade (diferenciada em graus e, também, em qualidade), na agenda das políticas de saúde, principalmente quando se trata da agenda local de saúde (Carneiro Junior; Silveira, 2003; Marsiglia e col., 2005).

A inclusão das diferenças nas sociedades complexas, por meio das políticas sociais, é uma questão essencial que levaria às chamadas políticas de integração (Castel, 1997). Há, portanto, que se pensar a inclusão como um complexo conjunto de idéias e práticas que tenha como pressuposto básico a diminuição das desigualdades sociais. Os segmentos cujas condições de vida estão muito aquém do mínimo necessário à manutenção de suas condições de saúde, como sói acontecer com a população em situação de rua, encontram-se em situações de vida tais que as respostas dadas pelas políticas, e consubstanciadas pelos serviços por meio de ações diretas, muitas vezes, não alcançam os efeitos planejados (Carneiro Junior e col., 1998). Assim, o eixo relacional estabelecido entre os serviços públicos de saúde e os segmentos vulneráveis assume um caráter de fragilidade, em decorrência da ausência de uma percepção mais clara e precisa dos reais problemas e das respostas mais efetivas que possam aliviar o sofrimento e fazer a manutenção de uma atenção à saúde integral, como preconizado pela Constituição Federal de 1988 (Brasil, 1988).

Segundo Castel (1997), as políticas de integração devem contemplar a compreensão das situações que geram diferenças e desigualdades acentuadas nas sociedades complexas. A ruptura da estrutura familiar e das redes de apoio social, a ausência ou a perda de redes comunitárias, o fim da condição salarial e do emprego estável são elementos que normalmente levam a processos, muitas vezes irreversíveis, de vulnerabilidade.

Nesse sentido, os processos que conduzem à vulnerabilidade, segundo Castel (1997), podem apresentarse nas seguintes instâncias processuais, denominadas pelo autor como zonas: a primeira, seria a zona de integração, que mantém os sujeitos vinculados por meio de inserções relacionais consolidadas e estáveis, incluindo a situação de trabalho estável; a segunda, zona de vulnerabilidade, cuja característica principal, por um lado, é a instabilidade no trabalho (que assu- me as características de ser temporário e instável) e, por outro, a fragilidade ou a ausência de recursos sociais que permitam oferecer suporte e cuidados às pessoas. Essa é uma instância que abrange grupos de famílias, ou sujeitos isolados, em situações incertas, cujo futuro não possa ser de maneira alguma pensado como um campo de certezas ou mesmo de possibilidades de experiências de vida menos sofridas. Esses fatores levam os sujeitos a procurarem situações de trabalho precárias, seja pelas condições objetivas do trabalho, que colocam em risco sua própria saúde, seja pelo caráter temporário das atividades e da remuneração, que quase sempre é ínfima diante de suas necessidades imediatas. Tem-se ainda a ausência ou a fragilidade de suportes sociais que permitam a manutenção e o fortalecimento de suas condições de vida.

Se os primeiros sinais de desintegração relacional têm inicio na zona de vulnerabilidade, o passo seguinte é a chamada desfiliação, cuja marca principal é a impossibilidade de participar de qualquer atividade produtiva e também de um isolamento relacional, que, aos poucos, vai lançando os sujeitos em trajetórias cada vez mais complexas, do ponto de vista de sua capacidade de resolução.

A exclusão dos diferentes grupos: familiar, quase sempre impulsionada pelo consumo exagerado de álcool e suas conseqüências nefastas; religioso, que normalmente agrega e cria um campo de sentidos à existência nos âmbitos individual e coletivo; comunitário, desenvolvido nas vizinhanças do bairro, do trabalho e dos grupos relacionais formados a partir dele - o informal, da roda de amigos e o formal, da organização sindical - lança os sujeitos à incerteza do futuro, levando à capacidade de reconquistar seu potencial para a manutenção e o desenvolvimento de relacionamentos.

Assim, torna-se essencial à produção de serviços em saúde a incorporação da visão de mundo desses segmentos sociais e não somente suas características sociodemográficas e epidemiológicas. No caso particular da população em situação de rua, a perda do potencial crítico de contestação e de cobrança das políticas sociais como direito de cidadania poderia determinar uma existência cuja sorte coloca-se subjugada à vida na metrópole.

Podem ser observados, no conjunto de membros representantes desse segmento social, que os proces- 
sos de exclusão são múltiplos e suas conseqüências têm quase sempre o mesmo fim: a vida torna-se solitária. O desamparo quase sempre predomina, salvo em casos de grupos que se formam e passam a freqüentar espaços públicos transformados em moradias. Adicionalmente, a exposição à violência - física e simbólica -, quase sempre produzida pelo próprio Estado, é freqüente e muitas vezes a resposta é o silêncio e a conformação. $O$ consumo de álcool cria um círculo vicioso: o sujeito sofre exclusão por beber constantemente e passa a ingerir álcool por ter sido excluído. Por fim, sofre comprometimentos à saúde física e mental, muitas vezes irreversíveis, determinando experiências de adoecimento freqüentes e complexas pelo conjunto de patologias a elas associadas (Snow; Anderson, 1998; Rosa, 2005).

Essa seria, portanto, a expressão maior da desfiliação. Uma ampla compreensão da situação enfrentada pelos moradores de rua normalmente não considera as conseqüências dessa desfiliação, sobretudo seus aspectos quase nunca objetivados, como o desenvolvimento de relações intersubjetivas, relações com o poder público por meio de seus equipamentos e aspectos subjetivos, que ampliem o campo de conhecimento das percepções e concepções destes sujeitos sobre saúde e doença (Carneiro Junior e col., 1998).

A inclusão social está inscrita no SUS em seus princípios de universalidade e de igualdade, inaugurando uma lógica pública na organização dos serviços e das ações de saúde, na perspectiva da integralidade do cuidado e da diminuição das desigualdades sociais.

A atenção integral e o cuidado dispensados aos grupos que sofrem as conseqüências dos processos de exclusão social devem rejeitar a visão de que as diferenças criadas, e normalmente incorporadas por meio do estigma e da rejeição, constituam barreiras às ações em saúde, enfim, a inclusão social, essencial à vida, deve prevalecer nas concepções e nas práticas dos serviços, produzindo ações que mantenham a saúde de forma integral e promovam a capacidade dos sujeitos na recuperação de sua autonomia e responsabilidade.

Para que isso ocorra, é necessário conceber e organizar processos de trabalho que dêem visibilidade a esses grupos sociais e incorporem no cotidiano das práticas sanitárias um certo "saber fazer" na atenção às demandas trazidas por esses indivíduos, articulando-os no conjunto de outras práticas sociais.

Nessa perspectiva, um dos níveis de atenção à saúde que oferece maior possibilidade de construção dessas práticas é a atenção primária à saúde, pois se constitui um importante espaço para a realização de processos de trabalho em saúde mais horizontais e está inserida, necessariamente, em um determinado território social.

\section{Atenção Primária à Saúde e Organização da Eqüidade no Acesso aos Serviços de Saúde}

De acordo com Merhy, “(...) a rede básica [atenção primária], diferentemente do hospital, apresenta a possibilidade de menos aprisionamento de suas práticas a um processo de trabalho médico restrito, ou mesmo circunscrito no tempo e no evento, podendo abrir-se, portanto, às mais distintas alternativas de constituição de novos formatos de produção das ações de saúde" (Merhy, 1997, p. 199).

De maneira geral, a atenção primária tem sido reconhecida mundialmente como uma importante tecnologia em saúde. A inserção desses serviços em um território determinado, permitindo que se reconheça e se estabeleça relações com a população de sua área e se articule com as diferentes instituições presentes nesse espaço geográfico, proporciona resultados efetivos no cuidado e na atenção integral à saúde (Starfield, 2002).

Esse entendimento é bastante promissor no caminho apontado anteriormente, pois permite nesse nível da atenção possibilidades de organizar ações mais flexíveis, que se adaptem às especificidades dos grupos sociais que demandem cuidados individuais e coletivos.

Nesse sentido, os serviços de atenção primária em saúde são campos férteis para se operar práticas que visem ao enfrentamento dos desafios da incorporação da heterogeneidade, das desigualdades sociais e das diferentes necessidades de saúde, particularmente, nas áreas centrais de grandes metrópoles, por meio da organização da eqüidade ao acesso a esses serviços (Carneiro Junior; Silveira, 2003).

Por sua vez, a eqüidade tem sido bastante discutida nos últimos anos, colocada na agenda pública dos 
vários setores sociais, particularmente o SUS. Todavia, há vários entendimentos sobre esse termo, que, para alguns, assume o mesmo entendimento de igualdade, expresso no capítulo da Saúde, na Constituição de 1988; para outros a eqüidade tem forte acento na justiça social, na busca da diminuição das desigualdades sociais; entretanto, tem sido cada vez mais presente nos estudos e nos documentos norteadores do SUS, mesmo que às vezes genéricos, o entendimento de que a eqüidade se refere a políticas que visem a redução das iniqüidades de saúde e de acesso aos serviços, isto é, reconhecem que há grupos sociais desprovidos de assistência à saúde, tornando-se vulneráveis aos processos de adoecimento e morte (Campos, 2006; Paim, 2006).

Campos (2006) alerta para a necessidade de se organizar práticas equânimes cotidianas e singulares nos serviços de saúde, pois, dessa maneira, as possibilidades de consolidação dos pressupostos do SUS seriam evidenciadas, transpondo para a micropolítica os ideários e o discurso que se têm concentrado na macropolítica do sistema de saúde brasileiro.

Uma discussão está em pauta é a necessidade de que a eqüidade em saúde seja incorporada pelos atores sociais envolvidos no controle social do SUS pois, assim, ela estaria mais orientada para a produção de sujeitos autônomos, comprometidos com essas práticas, garantindo sua consolidação no interior das práticas de saúde (Costa; Lionço, 2006).

De acordo com essa perspectiva, têm sido organizadas e sistematizadas as práticas de atenção primária à saúde, no Centro de Saúde Escola Barra Funda Dr. Alexandre Vranjac (CSEBF-AV). Desde 1996, a eqüidade no acesso aos serviços de saúde da área central da cidade de São Paulo orienta a construção de novas tecnologias em saúde, visando incluir os grupos em processo de exclusão social.

\section{Incorporação das Populações em Situação de Exclusão Social no CSEBF-AV}

O CSEBF-AV é um serviço de atenção primária da Irmandade da Santa Casa de Misericórdia de São Paulo, localizado na região central do município de São Paulo. Concebido e estruturado no final dos anos
1960, teve sua implementação pautada pelo modelo tecnológico da programação em saúde objetivando, principalmente, corresponder às políticas de extensão da cobertura da assistência médica a grupos populacionais "marginalizados" dos centros urbanos, na perspectiva do planejamento em saúde (Nemes, 1990).

A população da área de abrangência do CSEBF-AV constituía-se essencialmente de moradores domiciliados e trabalhadores do mercado formal e informal da região, que faziam uso do serviço através de consultas agendadas e, eventualmente, de atendimentos nãoagendados, denominados prontos-atendimentos.

A partir dos anos 1990, a região começa a sofrer mudanças importantes, com a chegada de empresas e intensificação da malha viária da cidade, ocasionando, em muitos aspectos, uma degradação do espaço urbano.

Nesse processo, o CSEBF-AV começou a se deparar com singulares grupos populacionais, que se traduziriam em desafios para os profissionais do serviço de saúde, como moradores de rua, que dificilmente se enquadravam nas propostas tradicionais de tratamento; profissionais do sexo, de baixo poder aquisitivo, que só podiam utilizar o serviço em períodos específicos; migrantes em situação irregular no país, sobretudo bolivianos, em precárias condições de moradia e trabalho (Marsiglia; Carneiro Junior, 1997).

A nova realidade resultou em uma reorganização tecnológica do serviço, contextualizado na construção do SUS, que deveria atender esse novo público e garantisse acesso a esses grupos populacionais de inserção desigual (Carneiro Junior; Silveira, 2003).

A concepção de eqüidade, com forte acento na estratégia da discriminação positiva, foi a resposta encontrada para garantir acesso diferenciado a essas populações que não se enquadravam na estruturação tradicional da unidade. Para os profissionais do CSEBFAV, a noção de excluídos sociais possibilitava a organização de perfis populacionais, em que processos de desvinculação social eram facilmente reconhecidos.

Os primeiros passos para buscar respostas adequadas eram, naquele momento, reconhecer esses grupos no interior do serviço, tornando-os visíveis na relação com as ações operadas, possibilitando, assim, uma reflexão com o conjunto dos profissionais sobre as nossas respostas e uma combinação com outras práticas e usuários. 
Desse modo, a melhor forma de entrada dessa população no serviço foi por meio do uso do pronto-atendimento, que foi estratégico para a vinculação da população e a oportunidade de fazer o maior número de abordagens possíveis no mesmo dia do atendimento, como consulta com saúde mental, serviço social, enfermagem, entre outras.

A identificação da situação de exclusão social no serviço é realizada por meio do preenchimento de um campo identificador de situação de matrícula, na ocasião de cadastramento na unidade. 0 campo identificador é preenchido com as seguintes informações: morador da área de abrangência do CSEBF-AV, morador fora da área de abrangência, morador de rua ou profissional do sexo; para o preenchimento desse campo é utilizado preferencialmente um comprovante de endereço. No caso de população em situação de rua, a situação de matrícula é confirmada com auto-declaração do usuário como morador de rua. Em relação às profissionais do sexo, para confirmar a situação de matrícula é necessária a entrega de filipeta de encaminhamento, distribuída nas atividades de campo realizadas pelo serviço.

Com esse processo, foi possível identificar alguns grupos populacionais que se tornaram visíveis e inclusos no cuidado à saúde: 1) a população em situação de rua; 2) o grupo das profissionais do sexo da região da Luz, que são diferenciadas de outras mulheres que trabalham na prostituição por sua condição socioeconômica, caracterizada por rendas extremamente baixas (Andrade; Silva, 1999); 3) os imigrantes bolivianos, 4) os moradores de favela.

\section{Os Grupos em Situação de Exclusão Social no CSEBF-AV em Números}

Após uma década de organização dos serviços oferecidos pelo CSEBF-AV, é possível apresentar um balanço da inclusão desses grupos, assim como algumas de suas características sociodemográficas.

No CSEBF-AV, estavam matriculados 21.928 indivíduos em junho de 2006. A distribuição segundo a situação de matrícula foi: $71 \%$ moradores da área, $8 \%$ trabalhadores da área, 10\% pessoas de fora de área, $7 \%$ população em situação de rua, 1 \% profissionais do sexo, e os demais em outras situações de matrícula. É interessante destacar que dos 15.575 moradores domiciliados $11 \%$ eram boliviano e $16 \%$ moradores de favelas. Essa distribuição pode refletir, portanto, a heterogeneidade da população dessa área de abrangência.

O reflexo do acesso a população em situação de exclusão social pode ser avaliado por meio da análise do processo de inclusão no serviço ao longo dos anos. Os indivíduos em situação de exclusão social iniciaram um processo mais acentuado de matrícula no serviço a partir de 1996, quando começaram a ocorrer atividades relacionadas tanto às buscas ativas de novos matriculados quanto ao treinamento da equipe para realização do acolhimento (Tabela 1). No período subseqüente, ocorreu um importante incremento no número de novas matrículas, em especial de moradores de favela e profissionais do sexo. Esse aumento pode ser decorrente da resposta dessas populações às ações desencadeadas pela equipe de saúde e, em especial, às atividades de busca ativa, que são realizadas em campo.

\section{Tabela I - Distribuição da população em situação de exclusão segundo o tipo de matrícula e o período de início do seguimento, CSEBF-AV, de 1968 a junho de 2006*}

\begin{tabular}{|l|c|c|c|c|c|c|c|c|}
\hline Situação de matrícula & \multicolumn{2}{|c|}{ Boliviano } & \multicolumn{3}{|c|}{ Morador de favela } & \multicolumn{2}{c|}{ Morador de rua } & \multicolumn{2}{c|}{ Profissionalde sexo } \\
\hline Período de matrícula & N & $\%$ & N & $\%$ & N & $\%$ & N & $\%$ \\
\hline $1968 / 1995$ & 69 & 4,1 & 15 & 0,6 & 3 & 0,2 & 0 \\
\hline $1996 / 2001$ & 223 & 13,1 & 1158 & 47,7 & 404 & 28,2 & 302 & 78,9 \\
\hline $2002 / 2006$ & 1410 & 82,8 & 1255 & 51,7 & 1024 & 71,6 & 81 & 21,1 \\
\hline Total & 1702 & 100,0 & 2428 & 100,0 & 1431 & 100,0 & 383 & 100,0 \\
\hline
\end{tabular}

* Dados de 2006 digitados até o mês de junho. 
Na Tabela 1, pode-se observar também que ocorreu uma redução no número de matrículas novas de profissionais do sexo no último período. Esse menor fluxo de matriculas desse grupo populacional devese à redução no número de atividades de campo no último ano. Outra possível explicação é a dificuldade de articulação de vários grupos de intervenção que atuam no território, governamentais e não-governamentais, que principalmente no último período avaliado desestimularam a procura de serviços de atenção primária da região, pois propiciaram alternativas de assistência em serviços especializados de atenção à população de profissionais do sexo. Em conseqüência desses resultados, estão sendo analisadas propostas para uma mudança da atuação no campo com a possível incorporação de um agente comunitário da saúde, e a implantação da estratégia de PSF nessa área de atuação.

O Gráfico 1 apresenta a distribuição de usuários segundo a sua situação de exclusão social no serviço de janeiro de 1996 a junho de 20o6. É interessante ressaltar que o serviço realmente conseguiu propiciar o acesso à população em situação de exclusão social da região central. Destaca-se a população em situação de rua, com 1.431 matriculados no serviço. Considerando que segundo estimativas da FIPE (2003) vivem hoje em toda a região central aproximadamente 10.0oo indivíduos em situação de rua, esse único serviço de atenção primária conseguiu matricular cerca de $14 \%$ dessa população. Os moradores de favelas, com 2.428 matriculados, são a maioria da população em situação de exclusão matriculada no serviço.

\section{Gráfico I - Distribuição de matriculados no CSEBF-AV segundo o tipo de exclusão social}

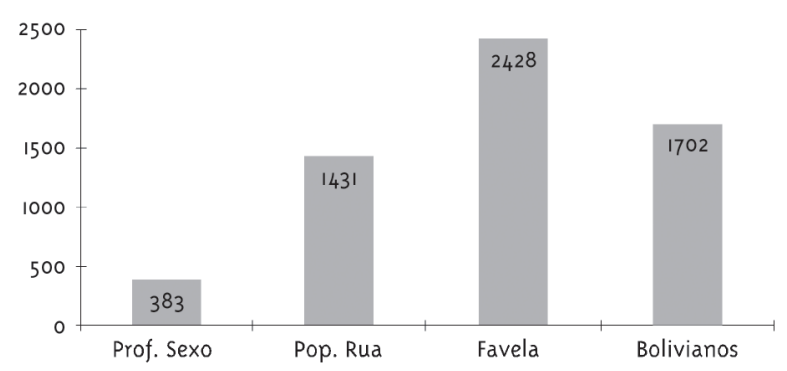

Ações que têm contribuído para a inclusão destes segmentos são: a flexibilização do horário de entrada na unidade, para os usuários em situação de exclusão, e o encaixe do atendimento sem necessidade de agendamento.

No início de 2001, a partir da implantação do PSF, as equipes iniciaram o cadastramento dos moradores da área e, dentre as várias repercussões para as ações em saúde, destaca-se a dos bolivianos, que representou para o serviço melhoria quanto ao controle dos cuidados junto às famílias, tendo destaque o trabalho dos agentes comunitários de saúde, como ferramenta fundamental nos processos de inclusão desse segmento no serviço. Assim, a partir de 2002, os bolivianos, que não procuravam anteriormente o serviço, mesmo sendo moradores do bairro, passaram a fazê-lo.

0 aumento do afluxo de moradores de rua no serviço, a partir de 2002 (Tabela 1), é uma importante resultado da implantação, na região central da cidade, de uma série de políticas públicas de inclusão dessa população em serviços de saúde, particularmente nos serviços de atenção primária. Essas transformações acabaram implementando o projeto "A Gente na Rua”, da Secretaria Municipal de Saúde de São Paulo, que constava da contratação de agentes comunitários de rua, preferencialmente ex-moradores de rua, para o cadastramento da população nos albergues da região ou na rua. Em 2004, esse projeto foi iniciado com um agente comunitário de saúde. Atualmente, são aproximadamente 31 agentes comunitários trabalhando na área central.

$A$ análise de dados referentes às matrículas dos usuários do CSEBF-AV, de 1996 até 2006, aponta para a existência de uma variação no processo de inclusão dessa população no período estudado, ou seja,, até dezembro de 2001 todas as matrículas eram realizadas na unidade, mas com a implantação das equipes do Programa de Saúde da Família (PSF), os cadastros de matrícula passaram a ser realizados nos domicílios, fato que garantiu uma extensão mais precisa dos registros dos moradores da área.

A Tabela 2 apresenta os dados relativos à escolaridade da população em situação de exclusão social, matriculada no CSEBF-AV. Pode-se observar que a maior freqüência de indivíduos que não sabem ler e escrever foi a de matriculados como moradores das 
áreas da favela. Considerando que o nível de escolaridade reflete a condição socioeconômica familiar e é, simultaneamente, variável proxy da inserção socioeconômica, podemos inferir que as condições socioeconômicas dos matriculados da favela são piores que as dos demais, com outras situações de exclusão social. Deve-se ressaltar que esse resultado confirma o achado de outros estudos conduzidos com a população em situação de rua (FIPE, 2003), refletindo a heterogeneidade dessa população com diferentes inserções socioeconômicas.

A distribuição dos matriculados segundo o sexo (Tabela 3) reflete as características das áreas de reali- zação das atividades do campo, porém não espelha a demanda geral do serviço, que apresenta número muito maior de mulheres matriculadas, no ano de 2006, segundo informações do SAFE (Sistema Ambulatorial de Faturamento e Estatística) do CSEBF-AV. Ressalta-se que, na região central, a população de rua masculina é bem superior que a feminina, isto é, 9o\% da população em situação de rua são homens (FIPE, 2003). É interessante observar que os serviços de atenção primária vêm atraindo mais mulheres em situação de rua do que o esperado, o que pode decorrer de ações de saúde da mulher organizadas nesses serviços, organizadas para atrair preferencialmente essa população.

\section{Tabela 2 - Distribuição da população em situação de exclusão matriculada segundo a escolaridade, CSEBF-AV, 2006*}

\begin{tabular}{|c|c|c|c|c|c|c|c|c|}
\hline \multirow{2}{*}{$\begin{array}{l}\text { Situação de matrícula } \\
\text { Escolaridade }\end{array}$} & \multicolumn{2}{|c|}{ Morador de favela** } & \multicolumn{2}{|c|}{ Bolivianos*** } & \multicolumn{2}{|c|}{ Moradores de rua\# } & \multicolumn{2}{|c|}{ Profissionais do sexo\#\# } \\
\hline & $\mathrm{N}$ & $\%$ & $\mathrm{~N}$ & $\%$ & $\mathrm{~N}$ & $\%$ & $\mathrm{~N}$ & $\%$ \\
\hline Não sabe ler/escrever & 921 & 38,5 & 180 & 10,7 & 256 & 18,4 & 16 & 4,4 \\
\hline Sabe ler e escrever & 37 & 1,5 & 41 & 2,3 & 38 & 2,6 & 4 & $\mathrm{I}, \mathrm{I}$ \\
\hline Menos de 8 anos de estudo & 593 & 24,8 & 346 & 20,8 & 582 & 41,5 & 49 & 13,9 \\
\hline 8 anos de estudo ou mais & 840 & 35,2 & 1108 & 66,2 & 525 & 37,5 & 284 & 80,6 \\
\hline Total & 2428 & 100,0 & 1702 & 100,0 & 1431 & 100,0 & 383 & 100,0 \\
\hline
\end{tabular}

* Dados de 2006 digitados até o mês de junho; ${ }^{* *} 27$ informações ignoradas; ${ }^{* * *} 37$ informações ignoradas; \#30 informações ignoradas; \#\#30 informações ignoradas.

\section{Tabela 3 - Distribuição da população em situação de exclusão social maticulada segundo o sexo, CSEBF-AV, 2006*}

\begin{tabular}{|l|c|c|c|c|c|c|c|c|}
\hline Situação de matrícula & \multicolumn{2}{|c|}{ Morador de favela } & \multicolumn{2}{|c|}{ Bolivianos } & \multicolumn{3}{c|}{ Situação de rua } & \multicolumn{2}{c|}{ Profissionais do sexo } \\
\hline Sexo & N & $\%$ & N & $\%$ & N & $\%$ & N & $\%$ \\
\hline Feminino & 1352 & 55,7 & 854 & 50,2 & 448 & 31,4 & 369 \\
\hline Masculino & 1076 & 44,3 & 848 & 49,8 & 980 & 68,6 & 14 & 3,6 \\
\hline Total & 2428 & 100,0 & 1702 & 100,0 & 1431 & 100,0 & 383 \\
\hline
\end{tabular}

* Dados de 2006 digitados até o mês de junho.

\section{Considerações Finais}

Nesses 10 anos de construção e organização dessa prática singular de saúde foram realizadas investigações científicas, articulados fóruns intersetoriais, treinamentos, cursos de extensão universitária e, tam- bém, grupos de estudos envolvendo profissionais do CSEBF-AV e de outros serviços. Fundamentalmente, os debates giraram em torno da noção de eqüidade em saúde, procurando-se construir maneiras de operacionalizá-la no serviço, para assim torná-la uma forma possível de promover a justiça social. 
O serviço incorporou como parte de sua tecnologia esse olhar específico, essas "portas de entrada", diferenciadas e o reconhecimento no cuidado à saúde de grupos diferenciados da população, em geral, necessitando de atenção particularizada, para diminuir os agravos à saúde, promovendo, desse modo, a eqüidade no acesso.

Como resultado desse esforço, podemos identificar o estabelecimento, no conjunto da rede local de saúde, do conceito de eqüidade e das estratégias particulares para sua viabilização no contexto das práticas de saúde, articuladas com outros setores sociais, particularmente, com a área de atuação da assistência social (Carneiro Junior e col., 200o).

Ainda hoje há desafios na organização dos serviços e questões conceituais na consolidação dessas práticas que precisam ser analisados no contexto dos processos de exclusão e inclusão social e nos modos de fazer o cuidado em saúde.

A integralidade no cuidado e a real participação desses sujeitos na produção da saúde são aspectos que devem ser adotados como preocupação no momento atual da organização tecnológica do CSEBF-AV, necessitando de investigações teóricas e metodológicas para o reconhecimento dessas questões, como também, para a promoção dessa reflexão no conjunto dos profissionais do serviço.

\section{Referências}

ANDRADE, M. C.; SILVA, J. L. DST em trabalhadoras do sexo: vulnerabilidade e exclusão. In: CONGRESSO PAULISTA DE SAÚDE PÚBLICA, 6., 1999, Águas de Lindóia. Livro de Resumos.... Águas de Lindóia: Associação Paulista de Saúde Pública, 1999. p. 19.

BRASIL. Constituição da República Federativa do Brasil, 1988. São Paulo: Imprensa Oficial do Estado, 1988.

CAMPOS, G. W. S. Reflexões temáticas sobre eqüidade e saúde: o caso do SUS. Saúde e Sociedade, São Paulo, v. 15, n. 2, p. 23-33, 2006.

CARNEIRO JUNIOR, N.; SILVEIRA, C. Organização das práticas de atenção primária em saúde no contexto dos processos de exclusão/inclusão social. Cadernos de Saúde Pública, Rio de Janeiro, v. 19, n. 6, p. 1827-1835, 2003.
CARNEIRO JUNIOR, N. et al. Serviços de saúde e população de rua: contribuição para um debate. Saúde e Sociedade, São Paulo, v. 7, n. 2, p. 47-62, 1998.

CARNEIRO JUNIOR, N. et al. A construção da eqüidade no acesso às políticas públicas: uma experiência de articulação intersetorial e interinstitucional na área central do município de São Paulo. Revista de Administração Pública, Rio de Janeiro, v. 34, n. 61, p. 95-104, 2000.

CASTEL, R. A dinâmica dos processos de marginalização: da vulnerabilidade à desfiliação. Caderno $C R H$, Salvador, n. 26/27, p. 19-40, jan./dez., 1997.

COSTA, A. M.; LIONÇO, T. Democracia e gestão participativa: uma estratégia para a eqüidade em saúde? Saúde e Sociedade, São Paulo, v. 15, n. 2, p. 47-55, 2006.

FIPE - Fundação Instituto de Pesquisa Econômica. Censo da população em situação de rua do município de São Paulo. São Paulo, 2003.

MARSIGLIA, R. M. G.; CARNEIRO JUNIOR, N. Condições de vida e saúde na região central de São Paulo. In: CONGRESSO BRASILEIRO DE SAÚDE COLETIVA, 5.; CONGRESSO PAULISTA DE SAÚDE PÚBLICA, 5., 1997, Águas de Lindóia. Livro de Resumos... Águas de Lindóia: Abrasco: APSP, 1997. p. 21.

MARSIGLIA, R. M. G.; SILVEIRA, C.; CARNEIRO JUNIOR, N. Políticas sociais: desigualdades, universalidade e focalização na saúde no Brasil. Saúde e Sociedade, São Paulo, v. 14, n. 2, p. 69-76, 2005.

MERHY, E. E. A rede básica como uma construção da saúde pública e seus dilemas. In: MERHY, E. E.; ONOCKO, R. (Org.). Agir em saúde: um desafio para o público. São Paulo: Hucitec; Buenos Aires: Lugar Editorial, 1997. p. 197-228.

NEMES, M. I. B. Ação programática em saúde: recuperação histórica de uma política de programação. In: SCHRAIBER, L. B. (Org.). Programação em saúde hoje. São Paulo: Hucitec, 199o. p. 65-116.

PAIM, J. S. Eqüidade e reforma em sistemas de serviços de saúde: o caso do SUS. Saúde e Sociedade, São Paulo, v. 15, n. 2, p. 34-46, 2006.

ROSA, C. M. M. Vidas de rua. São Paulo: Hucitec: Rede Rua, 2005. 
SNOW, D.; ANDERSON, L. Desafortunados: um estudo sobre o povo da rua. Petrópolis: Vozes, 1998.

STARFIELD, B. Atenção primária: equilíbrio entre necessidades de saúde, serviços e tecnologia. Brasília, DF: Unesco: Ministério da Saúde, 2002.

Recebido em: 04/09/2006

Aprovado em: 17/11/2006 